\title{
A Comparative Study to Noise Models and Image Restoration Techniques
}

\author{
Prabhishek Singh \\ Research Scholar \\ Department of Information \\ Technology \\ BBAU Lucknow
}

\author{
Raj Shree, PhD \\ Assistant Professor \\ Department of \\ Information Technology \\ BBAU Lucknow
}

\begin{abstract}
Image Restoration is one of area related to image processing which deals with restoring an original and sharp image from corrupted image using a mathematical degradation and restoration model. In this proposed work, a comparative study analysis of simple, fast technique is given to remove noise of an image which is mostly introduced due to environmental changes or due to other issues. Researchers focus on the noise issues that changes image pixels value either on or off. To get an enough efficient method to remove the noise from the images is a greater challenge for the researchers. Noise plays an important role in degrading the image at the time of capturing or transmission of the image. There are many algorithms and filtering techniques available which have their own assumptions, merits and demerits depending upon the prior knowledge of the noise. Image smoothening is one of the most significant and widely used procedure in the image processing. Here, apart from noise a model, the light is also thrown on comparative analysis of noise removal techniques is done. This paper will present the different noise types to an image models and investigating the various noise reduction techniques and their advantages and disadvantages and also it will help the new researchers to have the detailed and comparative knowledge regarding image restoration and all its associated details.
\end{abstract}

\section{Keywords}

Noise Models, Filters, Noise removal techniques, Image restoration

\section{INTRODUCTION}

Image restoration is an emerging field of image processing in which the focus is on recovering an original image from a degraded image. Image restoration can be defined as the process of removal or reduction of degradation in an image through linear or non-linear filtering. Degradation is usually incurred during the acquisition of the image itself. Just as in image enhancement, the ultimate goal in restoration is to improve an image. Enhancement is a subjective process while restoration is an objective process.

Restoration tries to reconstruct by using a priori knowledge of the degradation phenomenon. It deals with getting an optimal estimate of the desired result. Some restoration techniques are best achieved in the spatial domain, while there are some cases where frequency domain techniques are better suited [1].

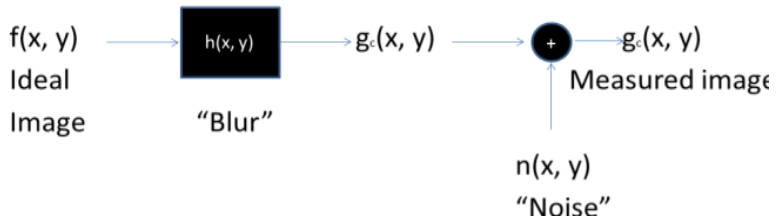

Degradation can be due to:

- Image sensor noise,

- Blur due miss focus,

- $\quad$ Noise from transmission channel etc.

The Purpose of smoothing is to reduce noise and improve the visual quality of the image. A variety of algorithms i.e. linear and nonlinear algorithms are used for filtering the images. Image filtering makes possible several useful tasks in image processing. A filtering technique can be applied to reduce the amount of unwanted noise in a particular image that is shown in Fig. 1 Another type of filter can be used to reverse the effects of blurring on a particular picture. Nonlinear filters have quite different behaviour as compared to linear filters. For nonlinear filters, the output or response of the filter does not follow the principles outlined earlier, particularly scaling and shift invariance. Moreover, a nonlinear filter can generate output that varies in a non-intuitive manner [2, 3, 4].

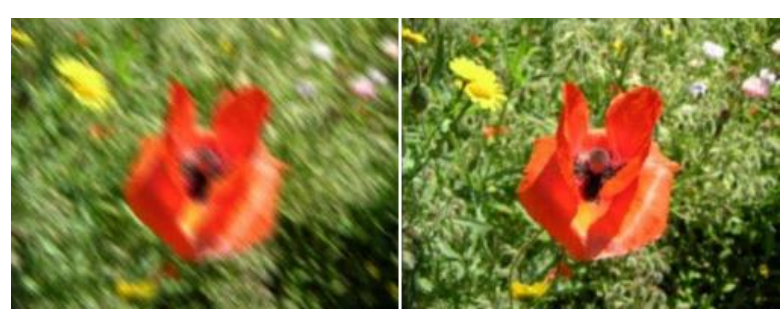

Defected image

Restored real image

Fig. 1. A Defected image and restored real image after applying filtering techniques [2]

Various application domains to Image restoration $[5,6]$ :

- Denoising and artifacts removal,

- sharpness, contrast, and resolution enhancement,

- Medical images (CT, MRI, ultrasound, etc.),

- Scientific explorations,

- Legal investigations,

- Film making and archival,

- Image and video (de-)coding

- Consumer photography,

- New applications: legacy materials, $\mathrm{HD} / 3 \mathrm{D} /$ mobile displays, web-scale data, etc.

There are so many causes of noise by which digital images are corrupted [7] such as malfunctioning pixels in camera sensors, faulty memory locations in hardware or transmission of 
image in a noisy channel and some other causes also [8]. Noise represents unwanted information which destroys the image quality [8]. It also affects the accuracy of many image processing applications such as image segmentation, image classification, edge extraction, image compression, etc. [8].

The restoration of image depends upon how much researchers know about the original image, information contains in the original image how much the image is degraded, reasons behind the degradations and how accurate our degradation models are [10] and with what accuracy it can be implemented [10].

\section{DEGRADATION AND RESTORATION MODEL}

The process of image restoration includes two sub processes. The first part deals with degrading the quality of the image by including blur and noise to an image and the second part deals with removing noise and blur from the degraded image and recovering the original image [9]. These two sub processes are named as degradation model and restoration model respectively. Both these are discussed below [9].

\subsection{Degradation Model}

Distortion is most of the time involved in recorded images. Distortion is mainly because of imperfections in the imaging system [10]. This problem can get extremely severe form due to random noise involved in the imaging.

In degradation model, the original image is blurred using degradation function and additive noise. The degraded image is described as follows:

$$
\mathrm{g}=\mathrm{h} * \mathrm{f}+\mathrm{n}
$$

In equation (1), $\mathrm{g}$ is the degraded image, $\mathrm{h}$ is the degradation function, $\mathrm{f}$ is an original image and $\mathrm{n}$ is the additive noise [12]. The degradation Model is structured as follows:

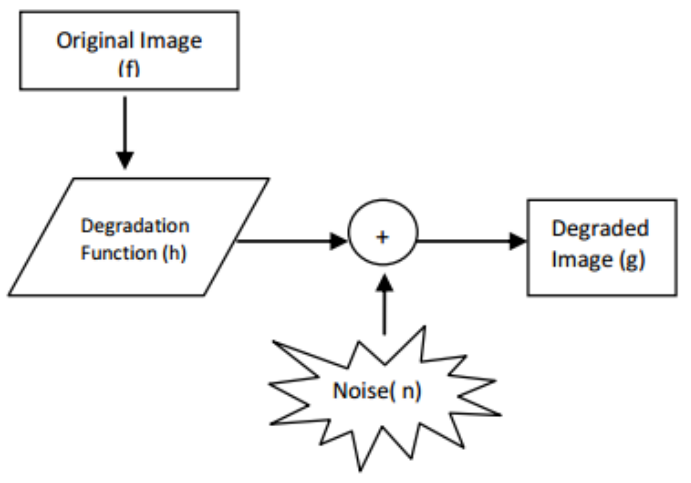

Fig. 2. Degradation Model [12]

\subsection{Restoration Model}

In Restoration model, the degraded image is reconstructed using restoration filters. In this process, noise and blur factor is removed and researchers get an estimate of the original image as a result of restoration. The closer the estimated image is to the original image the more efficient is our restoration filter. Figure 3 represents the structure of restoration model [11].

Fundamental concept

$\mathrm{g}(\mathrm{x}, \mathrm{y})=\mathrm{H}\{\mathrm{f}(\mathrm{x}, \mathrm{y})\}+\mathrm{\eta}(\mathrm{x}, \mathrm{y})$

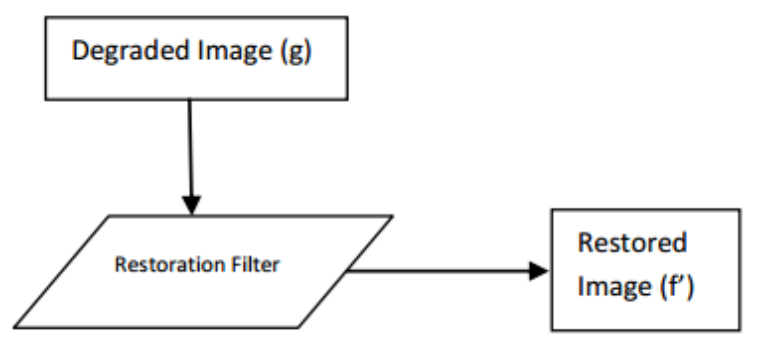

Fig. 3. Restoration Model [12]

\section{SOURCES OF NOISE}

Noise is normally a high frequency component and low pass filtering techniques is used to remove the noise [1]. Noise is introduced in the image at the time of image acquisition or transmission. There are different factors available for introducing noise in the image. The quantification of the noise will be decided by number of pixels corrupted in the image. The principal sources of noise in the digital image are [13]

- The imaging sensor may be affected by environmental conditions during image acquisition.

- Insufficient Light levels and sensor temperature may introduce the noise in the image.

- Interference in the transmission channel may also corrupt the image.

- If dust particles are present on the scanner screen, they can also introduce noise in the image.

- Blur due to miss-focus.

- Blur due to motion.

- Slow shutter speeds.

- High sensitivity modes (High ISO setting).

- Low light (night photos or dark scenes).

\section{NOISE MODELS}

Noise is the undesirable element produced in the image. During image possession or transmission, several factors are responsible for introducing noise in the image [13]. Depending on the type of disturbance, the noise can affect the image to different extent. Generally researchers keep focus on to remove certain kind of noise. Therefore researchers identify certain kind of noise and apply different algorithms to eliminate the noise. Image noise can be categorised as Impulse noise (Salt-and-pepper noise), Amplifier noise (Gaussian noise), Shot noise, Quantization noise (uniform noise), Film grain, on-isotropic noise, Multiplicative noise (Speckle noise) and Periodic noise [13].

Noise tells unwanted information in digital images [15]. Noise produces undesirable effects such as artefacts, unrealistic edges, unseen lines, corners, blurred objects and disturbs background scenes [15]. To diminish these undesirable effects, prior knowledge of noise models is required for further processing. Digital noise may arise from various kinds of sources such as Charge Coupled Device (CCD) and Complementary Metal Oxide Semiconductor (CMOS) sensors. In some sense, points spreading function (PSF) and modulation transfer function (MTF) have been used for timely, complete and quantitative analysis of noise models. Probability density unction (PDF) or Histogram is also used to design and characterize the noise models. 
Here researchers are trying to discuss few noise models, their types and categories in digital images $[14,15]$.

To understand the restoration process, it is imperative to understand the noise function $(\mathrm{x}, \mathrm{y})$. A good restoration system can be built if researchers could have realistic noise models. Noise in imaging systems is usually either additive or multiplicative. Noise is described by its probability characteristics.

\subsection{Gaussian Noise Model}

Good noise model due to its simplicity. They are very popular and are at times used when all other noise models fail.

It is also called as electronic noise because it arises in amplifiers or detectors [15]. Gaussian noise caused by natural sources such as thermal vibration of atoms and discrete nature of radiation of warm objects [16].

Gaussian noise generally disturbs the gray values in digital images. That is why Gaussian noise model essentially designed and characteristics by its PDF or normalizes histogram with respect to gray value. This is given as

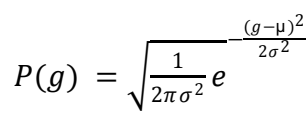

Where $\mathrm{g}=$ gray value, $\sigma=$ standard deviation and $\mu=$ mean. Generally Gaussian noise mathematical model represents the correct approximation of real world scenarios [15]. In this noise model, the mean value is zero; variance is 0.1 and 256 gray levels in terms of its PDF, which is shown in Fig. 4.

Due to this equal randomness the normalized Gaussian noise curve look like in bell shaped [15]. The PDF of this noise model shows that $70 \%$ to $90 \%$ noisy pixel values of degraded image in between and $\mu \sigma \mu \sigma-+$. The shape of normalized histogram is almost same in spectral domain [15].

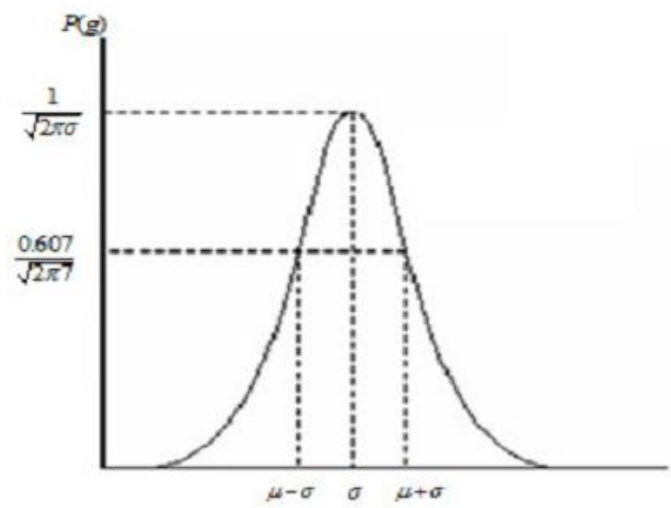

Fig. 4. PDF of Gaussian noise [15]

\subsection{White Noise}

Noise is essentially identified by the noise power. Noise power spectrum is constant in white noise. This noise power is equivalent to power spectral density function [15]. The statement "Gaussian noise is often white noise" is incorrect [14].

However neither Gaussian property implies the white sense. The range of total noise power is $-\infty$ to $+\infty$ available in white noise in frequency domain [15]. That means ideally noise power is infinite in white noise. This fact is fully true because the light emits from the sun has all the frequency components [15].

In white noise, correlation is not possible because of every pixel values are different from their neighbours. That is why autocorrelation is zero. So that image pixel values are normally disturbed positively due to white noise [15].

\subsection{Rayleigh Noise}

Unlike the Gaussian distribution, the Rayleigh distribution is not symmetric [1].

Rayleigh noise presents in radar range images. In Rayleigh noise, probability density function is given as [17].

$\mathrm{P}(\mathrm{g})=\left\{\begin{array}{cc}\frac{2}{b}(g-a) e^{\frac{-(g-a)^{2}}{b}} & \text { for } g \geq a \\ 0 & \text { for } g<a\end{array}\right.$

Where mean $\mu=\mathrm{a}+\sqrt{\frac{\pi b}{4}}$ and variance $\sigma^{2}=\frac{b(4-\pi)}{4}$ are given as, respectively.

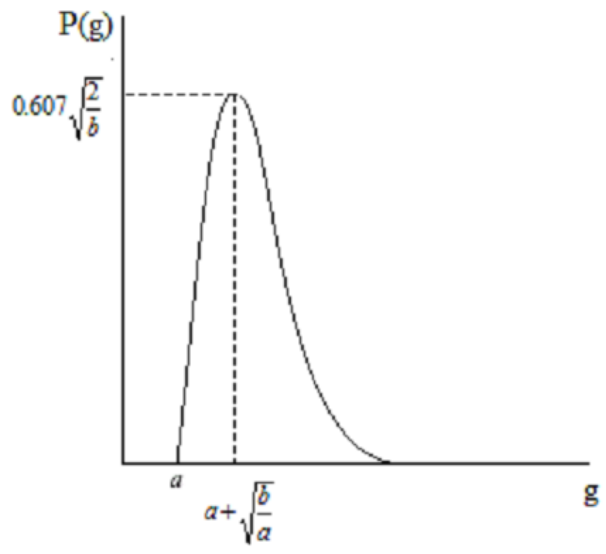

Fig. 5. Rayleigh distribution [15]

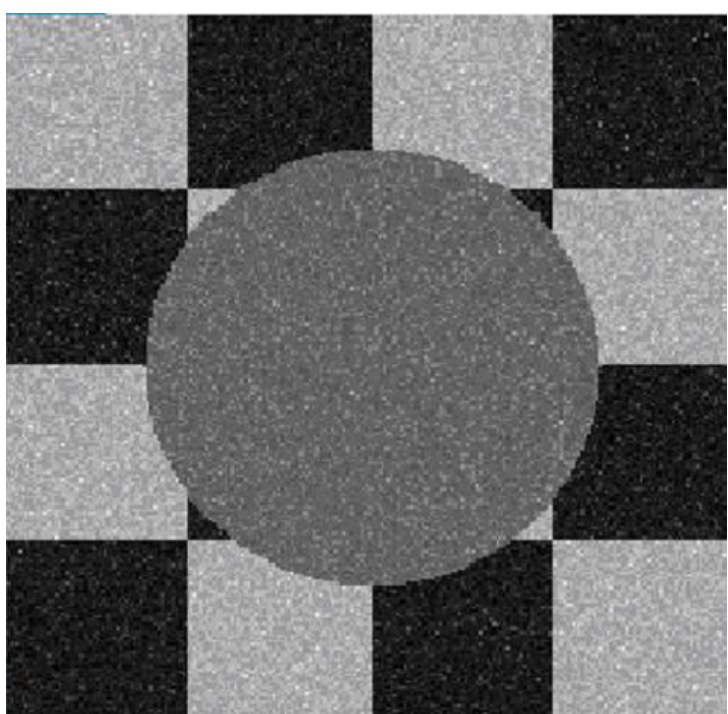

Fig. 6. Rayleigh Noise [17] 


\subsection{Brownian Noise (Fractal Noise)}

Colored noise has many names such as Brownian noise or pink noise or flicker noise or 1/f noise [15]. In Brownian noise, power spectral density is proportional to square of frequency over an octave i.e., its power falls on $1 / 4^{\text {th }}$ part $(6 \mathrm{~dB}$ per octave). Brownian noise caused by Brownian motion [15]. Brownian motion seen due to the random movement of suspended particles in fluid [15]. Brownian noise can also be generated from white noise, which is shown in Fig. 7.

However this noise follows non stationary stochastic process. This process follows normal distribution. Statistically fractional Brownian noise is referred to as fractal noise. Fractal noise is caused by natural process. It is different from Gaussian process [18-22].

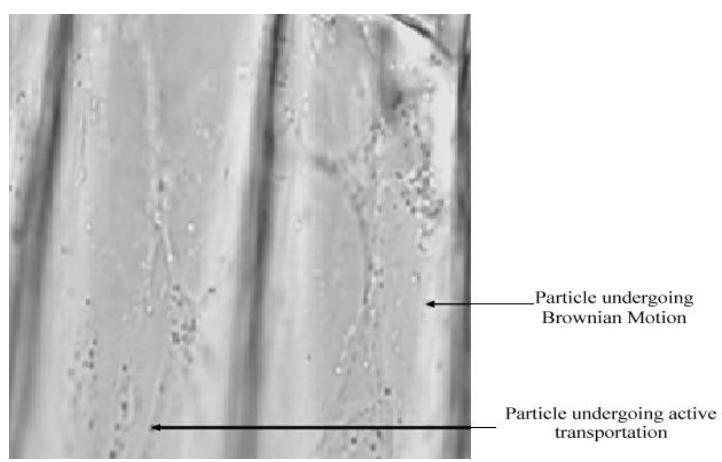

Fig. 7. Visualized vesicles in onion cells $(\mathrm{x} 20)$ form Brownian motion [23]

Although power spectrum of fractal noise, decaying continuously due to increase in frequency.

Fractal noise is almost singular everywhere. A fractional Brownian motion is mathematically represents as a zero mean Gaussian process $(\mathrm{BH})$ which is showing in equation (1) and equation (2), respectively [24].

$\mathrm{B}_{\mathrm{H}}(0)=0$

and expected value of fractional Brownian motion is

$\mathrm{E}\left\{\left|B_{H}(t)-B_{H}(t-\Delta)\right|^{2}\right\}=\sigma^{2}|\Delta|^{2 H}$

\subsection{Impulse Valued Noise (Salt and Pepper Noise)}

This noise appears as black and white dots on the entire image [1].

This is also called data drop noise because statistically its drop the original data values. This noise is also referred as salt and pepper noise [15]. However the image is not fully corrupted by salt and pepper noise instead of some pixel values are changed in the image. Although in noisy image, there is a possibilities of some neighbours does not changed [25-26].

This noise is seen in data transmission [15]. Image pixel values are replaced by corrupted pixel values either maximum 'or' minimum pixel value i.e., 255 'or' 0 respectively, if number of bits are 8 for transmission [15].

Let us consider $3 \times 3$ image matrices [15] which are shown in the Fig. 3. Suppose the central value of matrices is corrupted by Pepper noise. Therefore, this central value i.e., 212 is given in Fig. 8 is replaced by value zero [15].

In this connection, researchers can say that, this noise is inserted dead pixels either dark or bright. So in a salt and pepper noise, progressively dark pixel values are present in bright region and vice versa [27].

\begin{tabular}{|c|c|c|}
\hline 254 & 207 & 210 \\
\hline 97 & 212 & 32 \\
\hline 62 & 106 & 20 \\
\hline
\end{tabular}

Fig. 8. The central pixel value is corrupted by Pepper noise [15]

Inserted dead pixel in the picture is due to errors in analog to digital conversion and errors in bit transmission [15]. The percentage wise estimation of noisy pixels, directly determine from pixel metrics. The PDF of this noise is shown in the Fig.9.

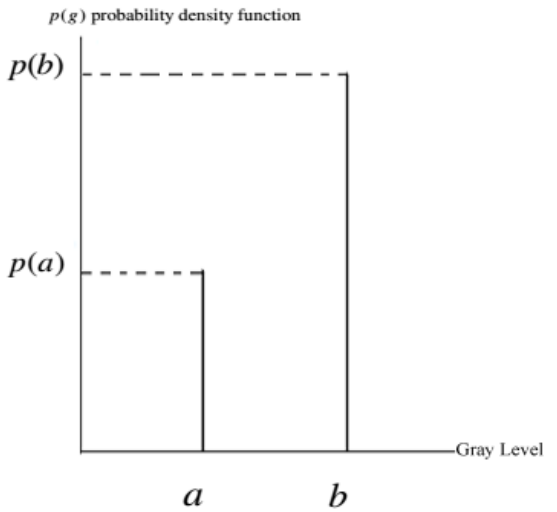

Fig. 9. The PDF of Salt and Pepper noise [15]

$\mathrm{P}(\mathrm{g})= \begin{cases}P_{a} & \text { for } g=a \\ P_{b} & \text { for } g=a \\ 0 & \text { otherwise }\end{cases}$

Fig. 4 shows the PDF of Salt and Pepper noise, if mean is zero and variance is 0.05 . Here researchers will meet two spike one is for bright region (where graylevel is less) called 'region a' and another one is dark region (where gray level is large) called 'region b' [15], researchers have clearly seen here the PDF values are minimum and maximum in 'region a' and 'region b', respectively [28]. Salt and Pepper noise generally corrupted the digital image by malfunctioning of pixel elements in camera sensors, faulty memory space in storage, errors in digitization process and many more.

\subsection{Periodic Noise (Sinusoidal Noise)}

It is a special kind of noise that cannot be eliminated in the spatial domain. Images are usually corrupted by the periodic noise due to electrical and electro mechanical interface during acquisition.

It is virtually impossible to remove periodic noise using spatial domain masks. The only way to eliminate them is by studying their response in the frequency domain [1].

\subsection{Speckle Noise}

This noise is multiplicative noise. Their appearance is seen in coherent imaging system such as laser, radar and acoustics etc. [15]. Speckle noise can exist similar in an image as Gaussian noise. Its probability density function follows gamma distribution, which is shown in Fig. 10 and given as in equation (1) [15].

$$
\mathrm{F}(\mathrm{g})=\frac{g^{\alpha-1} e^{\frac{-g}{a}}}{\alpha-1 ! a^{\alpha}}
$$




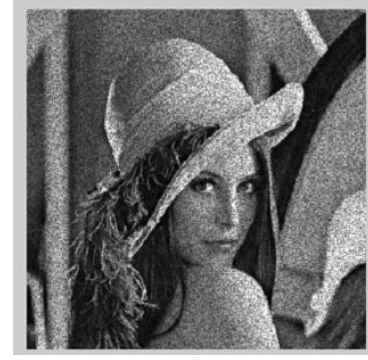

Fig. 9. Lena image [29] of Speckle noise with variance 0.04

\subsection{Quantization noise}

Quantization noise appearance is inherent in amplitude quantization process [15]. It is generally presents due to analog data converted into digital data. In this noise model, the signal to noise ratio (SNR) is limited by minimum and maximum pixel value, $\mathrm{P}_{\min }$ and $\mathrm{P}_{\max }$ respectively [15].

Quantization noise obeys the uniform distribution. That is why it is referred as uniform noise. Its PDF is shown in Fig.10 [15].

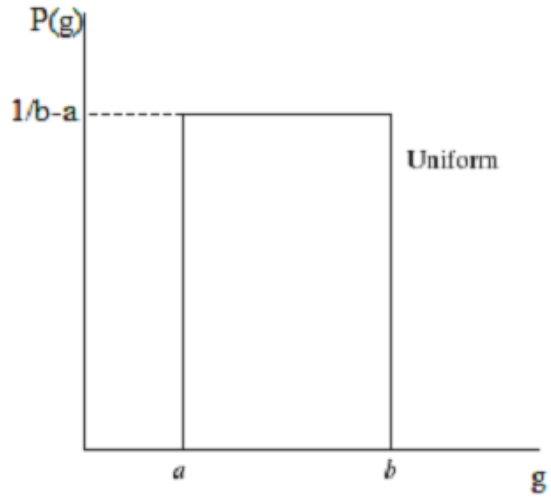

Fig.10. Uniform noise [15]

$$
\mathrm{P}(\mathrm{g})=\left\{\begin{array}{cc}
\frac{1}{b-a} & \text { if } a \leq g \leq b \\
0 & \text { otherwise }
\end{array}\right.
$$

and their mean $\mu=\frac{a+b}{2}$ and variance $\sigma^{2}=\frac{(b-a)^{2}}{12}$

\subsection{Photon Noise (Poisson Noise)}

The appearance of this noise is seen due to the statistical nature of electromagnetic waves such as

$\mathrm{x}$-rays, visible lights and gamma rays [15]. The x-ray and gamma ray sources emitted number of photons per unit time. These rays are injected in patient's body from its source, in medical $\mathrm{x}$ rays and gamma rays imaging systems [15]. These sources are having random fluctuation of photons. Result gathered image has spatial and temporal randomness. This noise is also called as quantum (photon) noise or shot noise [15]. This noise obeys the Poisson distribution and is given as

$$
\left.\mathrm{P}\left(f_{(p i)}\right)=\mathrm{k}\right)=\frac{\lambda^{k} e^{-\lambda}}{k !}
$$

\subsection{Structured Noise}

Structured noise is periodic, stationary or non-stationary and aperiodic in nature. If this noise is stationary, it has fixed amplitude, frequency and phase. Structured noise caused by interferences among electronic components [30]. Noise presents in communication channel are in two parts, unstructured noise (u) and structured noise (s). Structured noise is also called low rank noise [15]. In a signal processing, it is more advantagable (more realistic) to considering noise model in a lower dimensionality space [15].

Further, this model is mapped into full rank measurement space in physical system. So the researchers can conclude that in the measurement space, resulting noise has low rank and exhibits structure dependent on physical system[15].

Structured noise model is showing in equation (1) and equation (2), respectively [31].

$$
\begin{gathered}
\mathrm{y}_{(\mathrm{n})}=\mathrm{x}_{(\mathrm{n}, \mathrm{m})}+\mathrm{v}_{(\mathrm{n})} \\
\mathrm{y}_{(\mathrm{n})}=\mathrm{H}_{(\mathrm{n}, \mathrm{m})} * \mathrm{O}_{(\mathrm{m})}+\mathrm{S}_{(\mathrm{n}, \mathrm{t})} * \phi_{(\mathrm{t})}+\mathrm{v}_{(\mathrm{n})}
\end{gathered}
$$

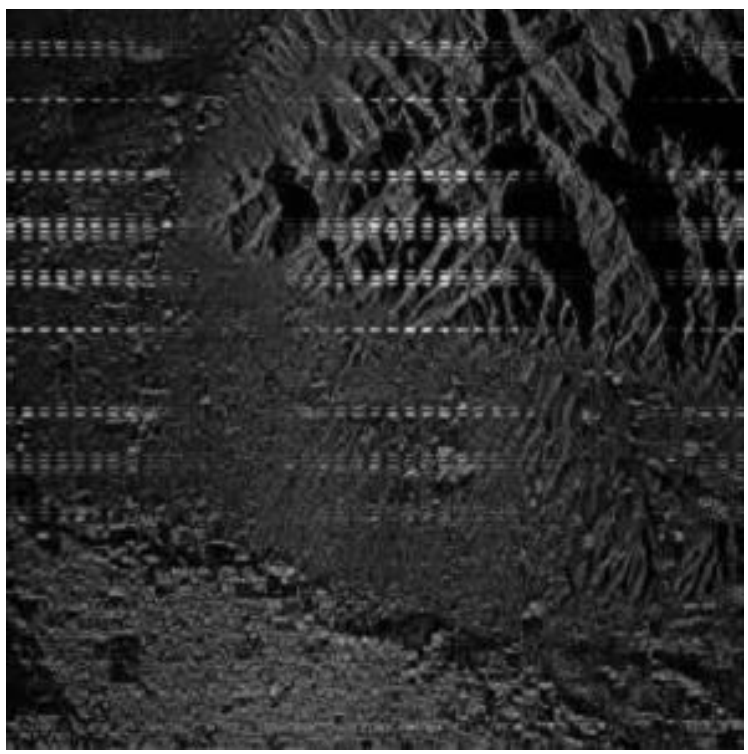

Fig. 11. Structured Noise (when noise is periodic and nonstationary) [15]

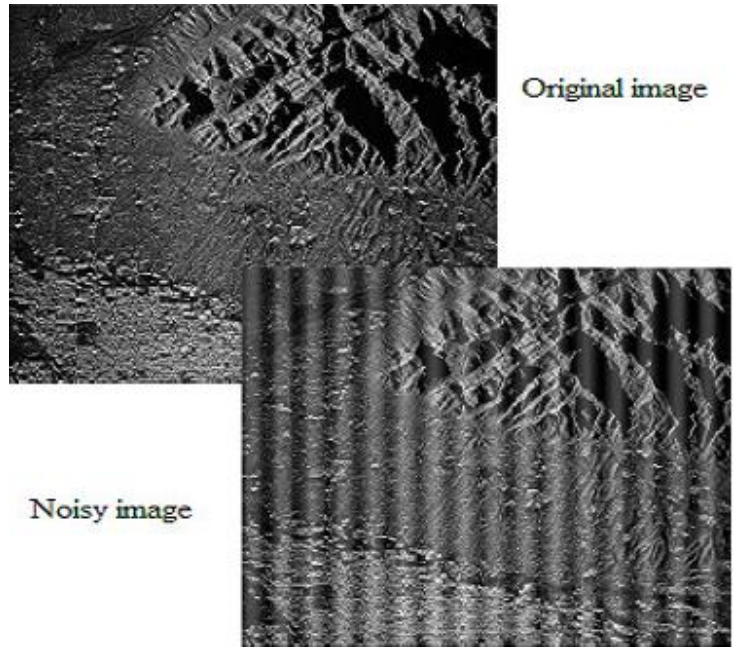

Fig.12. Structured Noise [31]

\subsection{Gamma Noise}

Gamma noise is generally seen in the laser based images. It obeys the Gamma distribution, which is shown in the Fig. 13 and given as [32-33] 


$$
P(g)= \begin{cases}\frac{a^{b} g^{b-1} e^{-a g}}{(b-1) !} & \text { for } g \geq 0 \\ 0 & \text { for } g<0\end{cases}
$$

Where mean $\mu=\frac{b}{a}$ and variance $\sigma^{2}=\frac{b}{a^{2}}$ are given as, respectively.

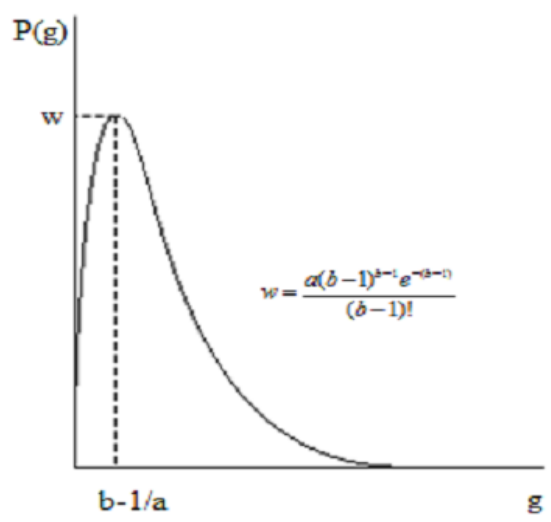

Fig. 13. Gamma distribution [15]

\section{IMAGE RESTORATION TECHNIQUES}

Image restoration techniques can be broadly classified into two types depending upon the knowledge of degradation.

\subsection{Deterministic method}

If the prior knowledge about degradation is known then the deterministic method of image restoration can be employed.

\subsection{Stochastic method}

If the prior knowledge about degradation is not known then the stochastic method of image restoration has to be employed.

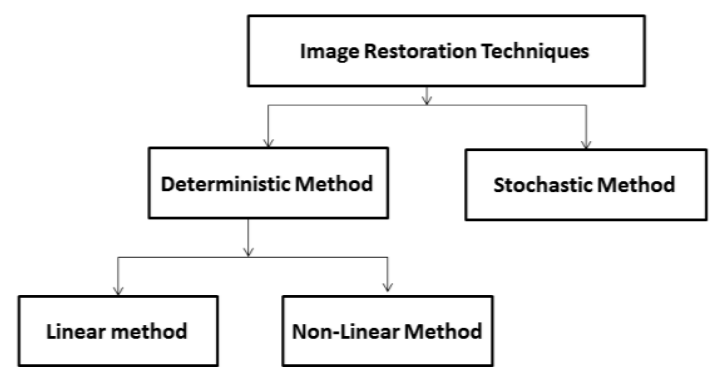

Image Degradation is a process by which image is blurred. The degradation is often modelled as a linear function which is often referred as point-spread function [8]. There are so many different causes of image degradation are: Improper opening \& closing of the shutter, atmospheric turbulence, miss the focus of the lens, relative motion between camera and object which causes motion blur [7].

\subsubsection{Linear Image Restoration Method}

If researchers know the exact PSF (Point Spread Function) model in the image degradation system then, the noise effect can be easily ignored [8]. The main drawback of this filter is, it will not perform well in the presence of noise. It will tend to amplify the noise [8].

The most common, simplest and fastest kind of filtering is achieved by linear filters [2]. The linear filter replaces each pixel with a linear combination of its neighbors and convolution kernel is used in prescription for the linear combination [34].

Linear filtering of a signal can be expressed as the convolution.

$$
y(t)=\int_{-\infty}^{\infty}(h(r) \cdot x(t-r) d r)
$$

of the input signal $x(n)$ with the impulse response $h(n)$ of the given filter [2], i.e. the filter output arising from the input of an ideal Dirac impulse. When researchers apply linear filtering then each pixel is replaced by linear combination of its neighbor [2].

\subsubsection{Non-Linear Image Restoration Method}

In recent years, a variety of non-linear median type filters such as rank conditioned, eighted median, relaxed median, rank selection have been developed to overcome the shortcoming of linear filter [32].

Non -Linear technique does not explicitly implement the inverse. It uses an iterative approach to produce a successive improvement to the restoration until a termination condition is reached. It can cope up with some missing frequency components, with non -Gaussian noise and non- negativity etc. [8].

\subsection{Briefly Discussed Image Restoration Techniques Inverse Filter}

This helps to get rid of the blurring (Deconvolution). The inverse filtering is a restoration technique for deconvolution, i.e., when the image is blurred by a known low pass filter, it is possible to recover the image by inverse filtering or generalized inverse filtering [16]. However, inverse filtering is very sensitive to additive noise. The approach of reducing one degradation at a time allows us to develop a restoration algorithm for each type of degradation and simply combine them [16]. The Wiener filtering executes an optimal trade-off between inverse filtering and noise smoothing. It removes the additive noise and inverts the blurring simultaneously [16].

\section{Pseudo-inverse filter}

It is a modified version of the inverse filter. It is actually a stabilized inverse filter. It gives much better results as comparison to inverse filter. It is too sensitive to noise.

\section{Wiener filter}

Most popular filter for restoration. It is not sensitive to noise. It exploits statistical properties of the image and can be used to restore the images in the presence of blur as well as noise.

The Wiener filtering is optimal in terms of the mean square error. In other words, it minimizes the overall mean square error in the process of inverse filtering and noise smoothing [16]. The Wiener filtering is a linear estimation of the original image. The approach is based on a stochastic framework [16]. The orthogonality principle implies that the Wiener filter in Fourier domain can be expressed as follows:

$\mathrm{W}(\mathrm{f} 1, \mathrm{f} 2)=\frac{H *(\mathrm{f} 1, \mathrm{f} 2) \mathrm{Sxx}(\mathrm{f} 1, \mathrm{f} 2)}{|H(\mathrm{f} 1, \mathrm{f} 2)|^{2} S x x(\mathrm{f} 1, \mathrm{f} 2)+S \eta \eta(\mathrm{f} 1, \mathrm{f} 2)}$

Where, $S_{x x}(f 1, f 2), S_{\eta \eta}(f 1, f 2)$ are respectively power spectra of the original image and the additive noise, and is the blurring filter. It is easy to see that the Wiener filter has two separate parts, an inverse filtering part and a noise smoothing part [16]. It not only performs the deconvolution by inverse 
filtering (high pass filtering) but also removes the noise with a compression operation (low pass filtering) [35].

\section{Constrained least-square filter.}

Bases optimality of restoration on a measure of smoothness. Seek minimum of criterion function

$\mathrm{C}=\sum_{x=0}^{M-1} \sum_{y=0}^{N-1}\left[\vec{V}^{2} f(x, y)\right]^{2} \quad$ (second derivative)

From the degradation system researchers get

$$
\begin{gathered}
g(x, y)=h(x, y) * f(x, y)+\eta(x, y) \\
g=H f+\eta(\text { vector-matrix form })
\end{gathered}
$$

The criterion function is subject to the constraint

$$
\|g-\tilde{H} \tilde{f}\|^{2}=\|\eta\|
$$

where ${ }^{\wedge} f$ is an estimate of the un-degraded image. The frequency domain solution thus becomes

$\mathrm{P}(\mathrm{u}, \mathrm{v})$ is the fourier transform of the Laplacian operator

\section{Median filter}

Median filter is a best order static, non- linear filter, whose response is based on the ranking of pixel values contained in the filter region [7]. Median filter is quite popular for reducing certain types of noise. Here the center value of the pixel is replaced by the median of the pixel values under the filter region $[7,36]$. Median filter is good for salt and pepper noise. These filters are widely used as smoothers for image processing, as well as in signal processing. A major advantage of the median filter over linear filters is that the median filter can eliminate the effect of input noise values with extremely large magnitudes [13]

\section{Mean filter}

Mean filter is an averaging linear filter [37]. Here the filter computes the average value of the corrupted image in a predecided area. Then the center pixel intensity value is replaced by that average value [13]. This process is repeated for all pixel values in the image.

Mean filters are classified as:

\begin{tabular}{|c|c|c|c|}
\hline Mean Filters & Working & Advantages & Disadvantages \\
\hline $\begin{array}{l}\text { Arithmetic } \\
\text { mean filters }\end{array}$ & $\begin{array}{l}\text {-Averaging mask used in spatial } \\
\text { domain enhancement. } \\
\text {-Computes the average value of a } \\
\text { neighborhood of a corrupted image } \\
\mathrm{g}(\mathrm{x}, \mathrm{y}) \text { and places the average } \\
\text { value at the center. }\end{array}$ & -Effective smoothing technique. & $\begin{array}{l}\text {-Loses details of the } \\
\text { image. }\end{array}$ \\
\hline $\begin{array}{l}\text { Geometric } \\
\text { mean filters }\end{array}$ & $\begin{array}{l}\text {-Center pixel of } \mathrm{g}(\mathrm{x}, \mathrm{y}) \text { is replaced } \\
\text { by the product of the pixels within } \\
\text { the neighborhood, raised to the } \\
\text { power } 1 /\left(\mathrm{M}^{*} \mathrm{~N}\right)\end{array}$ & $\begin{array}{l}\text {-Effective smoothing technique similar to } \\
\text { arithmetic mean filter. }\end{array}$ & $\begin{array}{l}\text {-Loses less detail as } \\
\text { comparison } \\
\text { arithmetic mean filter. }\end{array}$ \\
\hline $\begin{array}{l}\text { Harmonic } \\
\text { mean filters }\end{array}$ & $\begin{array}{l}\text {-The color value of each pixel is } \\
\text { replaced with the harmonic mean } \\
\text { of color values of the pixels in a } \\
\text { surrounding region. }\end{array}$ & $\begin{array}{l}\text {-Removes Gaussian type noise } \\
\text {-Salt noise (not pepper noise). } \\
\text {-Preserving edge features than the arithmetic } \\
\text { mean filter. } \\
\text {-The harmonic mean filter is very good at } \\
\text { removing positive outliers. }\end{array}$ & $\begin{array}{l}\text {-Unable to remove the } \\
\text { pepper noise. } \\
\text {-Some blurring effect. }\end{array}$ \\
\hline $\begin{array}{c}\text { Order } \\
\text { statistics filter }\end{array}$ & $\begin{array}{l}\text {-Order-Statistics filters are non- } \\
\text { linear filters whose response } \\
\text { depends on the ordering of pixels } \\
\text { encompassed by the filter area. }\end{array}$ & $\begin{array}{l}\text {-Effective in the case of Gaussian, speckle, } \\
\text { Salt and pepper and Poisson noises. }\end{array}$ & $\begin{array}{l}\text {-Blurs too much. } \\
\text {-Underlying stationary } \\
\text { assumption }\end{array}$ \\
\hline
\end{tabular}

$\hat{F}(u, v)=\left[\frac{H^{*}(u, v)}{|H(u, v)|^{2}+\gamma|P(u, v)|^{2}}\right] G(u, v)$

Table 1. Classification of Mean Filters

\section{Maximum Filter}

Applies a maximum filter to an image. The maximum filter is defined as the maximum of all pixels within a local region of an image [42].

The maximum filter is typically applied to an image to remove negative outlier noise [42].

$$
\check{\mathrm{f}}(\mathrm{x}, \mathrm{y})=\underset{(m, n) \in \mathrm{R}_{\mathrm{xy}}}{\max }\{\mathrm{g}(\mathrm{m}, \mathrm{n})\}
$$

\section{Minimum Filter}

Applies a minimum filter to an image. The minimum filter is defined as the minimum of all pixels within a local region of an image [43].

The minimum filter is typically applied to an image to remove positive outlier noise [43].

$$
\check{\mathrm{f}}(\mathrm{x}, \mathrm{y})=\underset{(\mathrm{m}, \mathrm{n}) \in \mathrm{R}_{\mathrm{xy}}}{\min }\{\mathrm{g}(\mathrm{m}, \mathrm{n})\}
$$

\section{Adaptive Filter}

These filters change their behaviour on the basis of statistical characteristics of the image region, encompassed by the filter region.BM3D is an adaptive filter. It is a nonlocal image modelling technique based on adaptive, high order group wise models [13].

This de-noising algorithm can be divided in three steps [3738]:

1. Analysis. Firstly similar image blocks are collected in groups. Blocks in each group are stacked together to form 
3-D data arrays, which are de-correlated using an invertible 3D transform

2. Processing. The obtained 3-D group spectra are filtered by hard thresholding.

3. Synthesis. The filtered spectra are inverted, providing estimates for each block in the group. These block-wise estimates are returned to their original positions and the final image reconstruction is calculated as a weighted average of all the obtained block-wise estimates [13]

\section{PERFORMANCE PARAMETERS}

Comparing restoration results requires a measure of image quality. Two commonly used measures are Mean-Squared
Error and Peak Signal-to-Noise Ratio [7]. The mean-squared error (MSE) between two images $\mathrm{g}(\mathrm{x}, \mathrm{y})$ and is:

$$
\mathrm{e}_{\mathrm{MSE}}=\frac{1}{M N} \sum_{n=1}^{M} \sum_{m=1}^{N}[\tilde{g}(n, m)-g(n, m)]^{2}
$$

One problem with mean-squared error is that it depends strongly on the image intensity scaling. A mean-squared error of 100.0 for an 8-bit image (with pixel values in the range 0255) looks dreadful; but a MSE of 100.0 for a 10-bit image (pixel values in $[0,1023]$ ) is barely noticeable $[13,44]$.

Table 2. Comparative analysis of Image Restoration/Filtering Techniques

\begin{tabular}{|c|c|c|}
\hline $\begin{array}{c}\text { Image Restoration/ } \\
\text { Filtering Techniques }\end{array}$ & Merits & Demerits \\
\hline Inverse Filter & $\begin{array}{l}\text {-Low pass filter } \\
\text {-Pass only low frequency parts of the image. } \\
\text {-Removes blurring effect }\end{array}$ & $\begin{array}{l}\text {-Restrict all the high frequency parts where the } \\
\text { noise dominates over the image. } \\
\text {-Results in amplification of noise. } \\
\text {-Sensitive to noise. }\end{array}$ \\
\hline Pseudo-inverse filter & $\begin{array}{l}\text {-Stabilised inverse filter. } \\
\text {-Shows much better results as compare to inverse } \\
\text { filters. } \\
\text {-Pass all the frequencies that satisfies the particular } \\
\text { threshold value } \varepsilon \text {. }\end{array}$ & -Sensitive to noise. \\
\hline Wiener filter & $\begin{array}{l}\text {-Popular filter used for restoration. } \\
\text {-Not sensitive to noise. } \\
\text {-Exploits the statistical properties of the image. } \\
\text {-Restore images in presence of blur as well as } \\
\text { noise. }\end{array}$ & $\begin{array}{l}\text {-Prior knowledge of the power spectral density } \\
\text { of the original image which is unavailable in } \\
\text { practice. }\end{array}$ \\
\hline $\begin{array}{l}\text { Constrained Least } \\
\text { Square Filter }\end{array}$ & $\begin{array}{l}\text {-Adds the Lagrange multiplier, } \lambda \text {, to control the } \\
\text { balance between noise artifacts and consistency } \\
\text { with the observed data }\end{array}$ & $\begin{array}{l}\text {-Requires some additional knowledge of the } \\
\text { original scene to be recovered }\end{array}$ \\
\hline Mean Filter & $\begin{array}{l}\text {-Simple method } \\
\text {-Intuitive method } \\
\text {-Easy to implement method of smoothing images }\end{array}$ & $\begin{array}{l}\text {-A single pixel with a very unrepresentative } \\
\text { value can significantly affect the mean value of } \\
\text { all the pixels in its neighborhood. } \\
\text {-When the filter neighborhood straddles an edge, } \\
\text { the filter will interpolate new values for pixels on } \\
\text { the edge and so will blur that edge. This may be } \\
\text { a problem if sharp edges are required in the } \\
\text { output. }\end{array}$ \\
\hline Median Filter & $\begin{array}{l}\text {-Preserve thin line edges, sharpness and fine details } \\
\text { from an input image. } \\
\text { - Both of the problems of mean filter are tackled by } \\
\text { the median filter }\end{array}$ & $\begin{array}{l}\text {-If a color image is corrupted by salt-and - } \\
\text { pepper noise, we cannot apply a median filter } \\
\text { directly. }\end{array}$ \\
\hline
\end{tabular}

Peak Signal-to-Noise Ratio (PSNR) avoids this problem by scaling the MSE according to the image range:

$$
\text { PSNR }=10 \log _{10}\left(\frac{L^{2}}{M S E}\right)
$$

where $S$ is the maximum pixel value. PSNR is measured in decibels $(\mathrm{dB})$. The PSNR measure is also not ideal, but is in common use. Its main failing is that the signal strength is estimated as $S^{\mathbf{2}}$, rather than the actual signal strength for the image. PSNR is a good measure for comparing restoration results for the same image, but between-image comparisons of PSNR are meaningless. One image with $20 \mathrm{~dB}$ PSNR may look much better than another image with $30 \mathrm{~dB}$ PSNR [44].

The MSE (mean square error) is defined as average squared difference between a reference image and a distorted image. It is calculated by the formula given below

$$
\mathrm{MSE}=\frac{1}{X Y}\left[\begin{array}{c}
X \\
\left.\sum_{i=1}^{X} \sum_{=1}^{X}(c(i, j)-e(i, j))^{2}\right]
\end{array}\right.
$$

$\mathrm{X}$ and $\mathrm{Y}$ are height and width respectively of the image. The $\mathrm{c}$ $(i, j)$ is the pixel value of the cover image and $e(i, j)$ is the pixel value of the embed image. [39]

The SNR (Signal to Noise ratio) measures the sensitivity of the imaging. It measures the signal strength relative to the background noise. It is calculated by the formula given below [40],

$$
\mathrm{SNR}_{\mathrm{db}}=10 \log _{10}\left(\frac{\text { Psignal }}{\text { Pnoise }}\right)
$$

The PSNR (peak signal to noise ratio) is used to determine the degradation in the embedded image with respect to the host image [41]. It is calculated by the formula as 


$$
\text { PSNR }=10 \log _{10}\left(\frac{L^{2}}{M S E}\right)
$$

$\mathrm{L}$ is the peak signal value of the cover image which is equal to 255 for 8 bit images [41].

The BER (bit error ratio) is the ratio that describes how many bits received in error over the number of the total bits received [41]. It is calculated by comparing bit values of embed and cover image.

$$
\mathrm{BER}=\frac{\mathrm{P}}{(\mathrm{H} * \mathrm{~W})}
$$

$\mathrm{H}$ and $\mathrm{W}$ are height and width of the watermarked image. $\mathrm{P}$ is the count number initialized to zero and it increments by one if there is any bit difference between cover and embed image [41].

\section{CONCLUSION}

Enhancement and restoration is necessary task in the digital image processing. There are various noise models available that can distort the images up to any extent. In order to restore such noisy images there are many image restoration and filtering techniques available that can recover the original image from the degraded image. In this paper maximum noise models are covered that gives a detailed understanding of all the perspectives of the noise including their advantages and disadvantages. Various sources of noises and applications of image restoration are also discussed. Apart from noise a detailed comparative study of image restoration and filtering techniques is also given that helps the new researchers to understand the various aspects of the image restoration that will create interest and lead them to work in specified hybrid technique.

\section{REFERENCES}

[1] Dhananjay K. Theckedath, Digital Image Processing (Using MATLAB Codes), Second Revised Edition: July 2013.

[2] Ruchika Chandel, Gaurav Gupta, Image Filtering Algorithms and Techniques: A Review, International Journal of Advanced Research in Computer Science and Software Engineering, Volume 3, Issue 10, October 2013

[3] Kailas, T.,"A view of three decades of linear filtering theory", Stanford University, Stanford, CA, USA.

[4] Datum. F," Aerospace and Electronic Systems Magazine", IEEE, 2005.

[5] http://www.journals.elsevier.com/signal-processing/callfor-papers/special-issue-on-image-restoration/

[6] Lexing Xie, Image Restoration, Lecture 7, March $23^{\text {rd }}$ 2009, EE4830 Digital Image Processing, http://www.ee.columbia.edu/ xlx/ee4830/

[7] "Digital Image Processing" R. C. Gonzalez and R. E. Woods, 2nd Ed., Englewood Cliffs, Nj: Prentice Hall, 2002.

[8] Pragati Agrawal, Jayendra Singh Verma , A Survey of Linear and Non-Linear Filters for Noise Reduction, International Journal of Advance Research in Computer Science and Management Studies, Volume 1, Issue 3, August 2013

[9] Rinku Kalotra , Sh. Anil Sagar, A Review: A Novel Algorithm for Blurred Image Restoration in the field of Medical Imaging, International Journal of Advanced
Research in Computer and Communication Engineering Vol. 3, Issue 6, June 2014

[10] Anamika Maurya, Rajinder Tiwari, A Novel Method of Image Restoration by using Different Types of Filtering Techniques, International Journal of Engineering Science and Innovative Technology (IJESIT) Volume 3, Issue 4, July 2014

[11] Amandeep Kaur Vinay Chopra, "Blind Image Deconvolution Technique for Image Restoration Using Ant Colony Optimization," International Journal of Computer Applications \& Information Technology Vol. I, Issue II, September 2012.

[12] Amandeep Kaur , Vinay Chopra, A Comparative Study and Analysis of Image Restoration

[13] Techniques Using Different Images Formats, "International Journal for Science and Emerging Technologies with Latest Trends" 2(1): 7-14 (2012).

[14] Rohit Verma, Dr. Jahid Ali, A Comparative Study of Various Types of Image Noise and Efficient Noise Removal Techniques, International Journal of Advanced Research in Computer Science and Software Engineering, Volume 3, Issue 10, October 2013.

[15] Dougherty G. (2010) "Digital Image Processing for Medical Applications," second ed., Cambridge university press.

[16] Ajay Kumar Boyat, Brijendra Kumar Joshi, A REVIEW PAPER: NOISE MODELS IN DIGITAL IMAGE PROCESSING, Signal \& Image Processing: An International Journal (SIPIJ) Vol.6, No.2, April 2015.

[17] Boyat, A. and Joshi, B. K. (2013) "Image Denoising using Wavelet Transform and Median Filtering', IEEE Nirma University International Conference on Engineering," Ahemdabad.

[18] Kamboj P. \& Rani V., (2013) "A Brief study of various noise models and filtering techniques," Journal of Global Research in Computer Science, Vol. 4, No. 4.

[19] Catipovic M. A., Tyler P. M., Trapani J. G., \& Carter A. R., (2013) "Improving the quantification of Brownian motion," American Journal of Physics, Vol.81 No. 7 pp. 485-491.

[20] Bhattacharya J. K., Chakraborty D., \& Samanta H. S., (2005) "Brownian Motion - Past and Present," Cornall university library. arXiv:cond-mat/0511389

[21] Radenovic A., "Brownian motion and single particle tracking," Advanced Bioengineering methods laboratory, Ecole polyteachenique federal de Lausanne.

[22] Peidle J., Stokes C., Hart R., Franklin M., Newburgh R., Pahk J., Rueckner W. \& Samuel AD, (2009) "Inexpensive microscopy for introductory laboratorycourses," American Journal of Physics Vol. 77 pp. 931-938.

[23] Nakroshis P., Amoroso M., Legere J. \& Smith C., (2003) "Measuring Boltzmann's constant using video microscopy of Brownian motion," American Journal of Physics, Vol. 71, No. 6, pp. 568-573. 
[24] Radenovic A., "Brownian motion and single particle tracking," Advanced Bioengineering methods laboratory, Ecole polyteachenique federal de Lausanne.

[25] Astola J. \& Kuosmanen P. (1997) "Fundamentals of nonlinear digital filtering," CRC Press, Boca Raton.

[26] Chabay R. W., \& Sherwood B. A., (2009) "Matter and Interactions," 3rd edition, John Wiley and Sons.

[27] Joshi, A., Boyat, A. and Joshi, B. K. (2014) "Impact of Wavelet Transform and Median Filtering on removal of Salt and Pepper noise in Digital Images," IEEE International Conference on Issues and Challenges in Intelligant Computing Teachniques, Gaziabad.

[28] Hosseini H. \& Marvasti F., (2013) "Fast restoration of natural images corrupted by high-density impulse noise," EURASIP Journal on Image and Video Processing. doi:10.1186/1687-5281-2013-15

[29] Koli M. \& Balaji S., (2013) "Literature survey on impulse noise reduction," Signal \& Image Processing : An International Journal (SIPIJ) Vol.4, No.5.

[30] Zhang L., Dong W., Zhang D. \& Shi G. (2010) “Two stage denoising by principal component analysis with local pixel grouping," Elsevier Pattern Recognition, Vol. 43, Issue 4, pp. 1531-1549.

[31] Behrens R. T. (1990) "Subspace signal processing in structured noise," Thesis, Faculty of the Graduate School of the University of Colorado, the degree of Doctor of Philosophy, Department of Electrical and Computer Engineering.

[32] Schowengerdt R. A. (1983) "Techniques for Image Processing and classifications in Remote Sensing," First Edition Academic Press.

[33] Kamboj P. \& Rani V., (2013) "A Brief study of various noise models and filtering techniques," Journal of Global Research in Computer Science, Vol. 4, No. 4.

[34] T. Chhabra, G. Dua and T. Malhotra (2013) "Comparative Analysis of Denoising Methods in CT
Images" International Journal of Emerging Trends inElectrical and Electronics, Vol. 3, Issue 2.

[35] Alexei Lufkin," Tips\& Tricks: Fast Image Filtering Algorithms"Moscow State University, Moscow, Russia.

[36] http://www.owlnet.rice.edu/ elec539/Projects99/BACH/ proj2/wiener.html

[37] D. Maheswari et. al. NOISE REMOVAL IN COMPOUND IMAGE USING MEDIAN FILTER. (IJCSE) International Journal on Computer Science and Engineering Vol. 02, No. 0 4, 2010, 1359-1362

[38] K. Dabov, A. Foi, V. Katkovnik, and K. Egiazarian, "Image denoising by sparse 3D transform-domain collaborative filtering," IEEE Trans. Image Process., vol. 16, no. 8, pp. 2080-2095, Aug. 2007.

[39] Aram Danielyan, Vladimir Katkovnik, and Karen Egiazarian, Senior Member, IEEE "BM3D frames and variational image deblurring", Image Processing, IEEE Transactions on (Volume:21, Issue: 4 ), ISSN: $1057-$ 7149

[40] Amit Kumar Singh, Nomit Sharma, Mayank Dave, Anand Mohan, -A Novel Technique for Digital Image Watermarking in Spatial Domainll, 2012 2nd IEEE International Conference on Parallel, Distributed and Grid Computing.

[41] http://en.wikipedia.org/wiki/Signal_to_noise_ratio_(imag ing)

[42] Amit Kumar Singh, Nomit Sharma, Mayank Dave, Anand Mohan, -A Novel Technique for Digital Image Watermarking in Spatial Domainll, 2012 2nd IEEE International Conference on Parallel, Distributed and Grid Computing.

[43] http://www.digimizer.com/manual/m-imagefiltermax.php

[44] http://www.digimizer.com/manual/m-imagefiltermin.php

[45] http://homepages.inf.ed.ac.uk/rbf/CVonline/LOCAL_CO PIES/VELDHUIZEN/node18.html 